\title{
Precise Modelling of Switching and Conduction Losses in Cascaded H-Bridge Multilevel Inverters
}

\author{
BasemAlamri \\ School of Engineering and Design, \\ Brunel University, \\ Basem.Alamri@brunel.ac.uk
}

\author{
Mohamed Darwish \\ School of Engineering and Design, \\ Brunel University, \\ Mohamed.Darwish@brunel.ac.uk
}

\begin{abstract}
Nowadays, voltage source multilevel inverters are being used extensively in industry due to its many advantages ,compared to conventional two level inverters, such as higher output voltage at low switching frequency, low voltage stress(dv/dt), lower total harmonic distortion (THD), less electro-magnetic interference (EMI), smaller output filter and higher fundamental output. However, the evaluation of multilevel inverter losses is much more complicated compared to two level inverters. This paper proposes an on-line model for precise calculation of conduction and switching losses for cascaded h-bridge multilevel inverter. The model is simple and efficient and gives clear process of loss calculation. A singlephase 7-level cascaded h-bridge with IGBT's as switching devices has been used as a case study of the proposed model. The inverter has been controlled using selective harmonic elimination in which the switching angles were determined using the Genetic Algorithm (GA). MATLAB-SIMULINK is used for the modelling and simulation.
\end{abstract}

Index Terms-Cascaded H-Bridge Multilevel Inverter (CHBMLI), Conduction Losses, Switching Losses, Selective Harmonic Elimination (SHE).

\section{INTRODUCTION}

In the last decade, the use of multilevel inverters has been grown numerously in most of the industrial power applications. It is hard to connect one power semiconductor switch directly in utility applications that requires medium voltage and high power level. Hence, the concept of multilevel inverters came as an alternative to conventional inverters in such applications. The multilevel inverters became very attractive as they produce low harmonic component at low switching frequency. In addition, they result in lower losses, lower blocking voltage of switching devices, and low electromagnetic interference (EMI) [1]. There are basically three main commercial topologies of multilevel voltage-source inverters well placed in industry which are: cascaded H-bridge (CHB-MLI), neutral-point clamped (NPC-MLI) and flying capacitor (FC-MLI). Among these topologies, the CHB-MLI is very widely used in industry for high power applications. It is used in high voltage and high power levels and it requires small number of devices, consequently, it has a reliable modular structure compared to the NPC-MLI and FC-MLI.

For power system planning and multilevel inverter design, accurate calculation of inverter power loss is of great importance to evaluate system efficiency, reliability and system operating cost. The design engineer should calculate the inverter losses precisely. In fact, loss evaluation in multilevel inverter is not an easy task and much more challenging compared to conventional two level inverters.
This is mainly because that the current differ in each power switch in the inverter. This paper focuses on a precise modelling of switching and conduction losses in CHB-MLI. Different methods were suggested in literatures to calculate the power loss in multilevel inverters. Some of these methods are based on online calculation from the simulated circuit and some are based on deep mathematical analysis and evaluation. In [2], each IGBT was modelled by characteristic curves using curve fitting exponential equations as a function of load current. In [3], a general scheme for calculating switching and conduction losses of power semiconductors in numerical circuits has been proposed. The model can be used online on the circuit simulation. Switching functions have been used in [4] to model the inverter losses for three phase nine level cascaded h-bridge inverter in which the load was assumed to be mixed RL load and the modulation index was 0.85. All the previous papers used online modelling for calculating the losses by applying curve fitting to characterize the IGBT based on the datasheet. On the other hand, in [5], [6] and [7], the losses of multilevel inverter have been calculated based on mathematical model in which the voltage across the switch as modelled by a threshold voltage and a series resistance.

This paper implement a model for calculating conduction and switching losses in CHB-MLI based on the method applied in [3] with little modification to be applied for multilevel inverters. A very clear and efficient procedure is to be explained in details which should serve as a guide in loss evaluation for multilevel inverters. The proposed modelling will be based on online simulation in which the inverter losses are calculated precisely with much less computational efforts. Basically, there are four types of losses in multilevel inverters which are: Conduction loss, Switching loss, OFF-state loss and Gate loss. The OFF-state and Gate losses are very small and normally neglected. Hence, in this paper only conduction and switching losses are considered throughout the analysis.

As it is most widely used in medium voltage, high power applications, the CHB-MLI has been considered for the analysis with IGBT's as power switching devices. First, Selective Harmonic Elimination (SHE) technique is to be performed to determine the switching angles which give minimum harmonic distortion. Genetic Algorithm (GA) optimization method is used for solving the system of transcendental equations. Then, simulation is conducted to evaluate the conduction and switching losses based on the proposed modelling. Curve fitting equations are implemented to model the power switch mathematically as per the datasheet. 


\section{CASCADED H-BRidge MUlTiLEVEl INVERTER (CHB-MLI)}

While Diode-Clamped (DC-MLI) and Flying Capacitor (FC-MLI) are widely used for industrial medium voltagehigh power applications when just low number of levels (typically three) is required, Cascaded H-Bridge inverters (CHB-MLI) are most suitable for high voltage-high power, HVDC utility applications. Mainly due its modular structure which can be extended for high number of levels with no much complexity. Furthermore, with CHB-MLI, higher power and voltage capability can be achieved at lowest number of required devices compared to DC-MLI and FCMLI. The CHB-MLI uses series connection of single phase hbridge inverters with separate dc sources. The main idea is that each bridge cell will generate three different voltages and the output waveform can be synthesized by the sum of the voltages generated by each cell. The separate dc sources might be solar panel PV cells or fuel cells.[1]

In this paper, a 7 Level CHB-MLI has been chosen to be investigated. The circuit layout for single phase 7-Level CHB-MLI is shown in Figure (1). The switching devices have been selected to be of IGBT type FZ1500R33HL3, which has a blocking voltage capability of $3.3 \mathrm{kV}$, and a maximum forward current of $1500 \mathrm{~A}$ and its shown in Figure(2). Datasheet for this type IGBT is given in [9]. Typical applications of this IGBT are: Chopper Applications, Medium Voltage Converters, Motor Drives, Traction Drives, UPS Systems and Wind Turbines [9]. Each cell is connected to a dc link supply of $100 \mathrm{~V}$. Modulation index of (0.8) was used throughout the analysis. The inverter has been modelled in MALTAL-SIMULINK with main objective of precisely calculating the conduction and switching losses of the inverter.

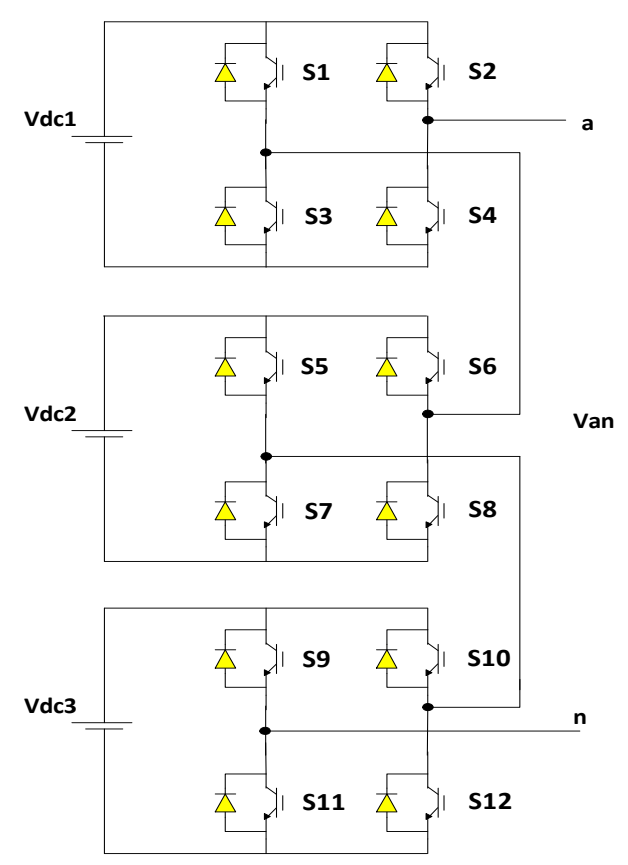

Fig. 1. Single-phase 7-level cascaded h-bridge inverter circuit layout.

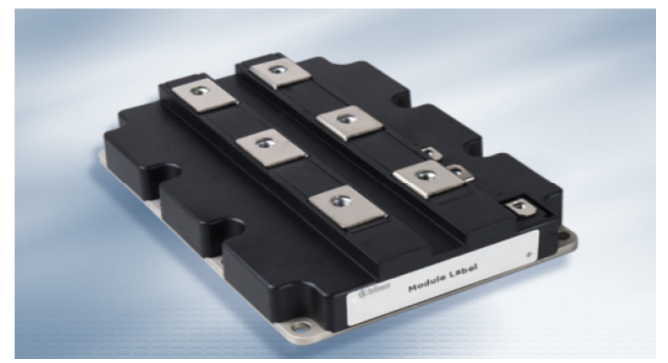

Fig. 2.IGBT module FZ1500R33HL3 typical appearance [9].

\section{Selective Harmonic Elimination (SHE)}

To control the output of voltage waveform in multilevel inverters, different modulation techniques have been applied. These control techniques are classified mainly based on the switching frequency into low or high switching techniques. Space Vector Control (SVC) and Selective Harmonic Elimination (SHE) are low switching techniques in which the active power switch is commutated only one or two times within one cycle. On the other hand, various PWM are used for high switching techniques in which the power switch is switched many times within a cycle [1]. In this analysis, SHE has been proposed for controlling the inverter as this technique has lower switching losses and less EMI because of its low switching [4]. In addition, it can eliminate the dominant low order harmonic and hence minimize the size of the required filter at the inverter output.

SHE uses pre-defined switching angles to form the desired multilevel fundamental voltage and eliminate the predominant low order harmonics which results in minimizing the total harmonic distortion (THD). The switching angles are precalculated off-line and hence this is considered open loop control technique. Figure (3) shows the stepped-voltage waveform for 7-level CHB-MLI. It is clear that, there are 3 switching angles which can be pre-calculated in this case.

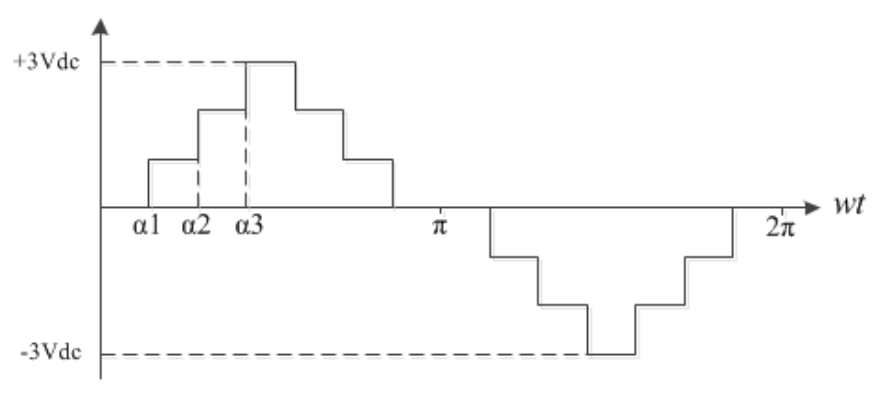

Fig. 3. Stepped-voltage waveform for 7-level inverter. 
Applying Fourier's expansion, the stepped voltage wave form can be expressed in sum of sine and cosine periodic signals and a constant. The signal consists of odd and even harmonics. Due to the quarter symmetry of the waveform, the even harmonics and the dc constant are cancelled. Hence, only odd harmonics are considered. For balanced three phase systems all triplen harmonics are zero. Generally, The output voltage waveform can be written as:

$v_{a n}(w t)=$
$\sum_{k=1,3,5, \ldots \ldots .}^{\infty} \frac{4 V_{d c}}{k \pi}\left[\cos \left(k \propto_{1}\right)+\cos \left(k \propto_{2}\right) \ldots . .+\cos \left(k \propto_{s}\right)\right] \sin (k w t)$

Where $(\mathrm{S})$ is the number of H-bride cells of the inverter.

It is clear from Figure (3) that all switching angles are less than $90^{\circ}$, and are all in ascending order. In 7-level CHB-MLI case,

$$
\propto_{1}<\propto_{2}<\propto_{3}<90^{\circ}
$$

And it is possible to eliminate the $5^{\text {th }}$ and $7^{\text {th }}$ harmonic by solving the following system of non-linear equations where $(m i)$ is the modulation index.

$$
\begin{aligned}
& \cos \propto_{1}+\cos \propto_{2}+\cos \propto_{3}=3 m_{i} \\
& \cos 5 \propto_{1}+\cos 5 \propto_{2}+\cos 5 \propto_{3}=0 \\
& \cos 7 \propto_{1}+\cos 7 \propto_{2}+\cos 7 \propto_{3}=0
\end{aligned}
$$

Newton-Raphson iterative method has been applied to solve such system in practice. Key issue is that when the inverter level gets higher, it becomes more difficult to get to the solution. In addition, it requires good initial guessed values of the switching angles. In this paper, Genetic Algorithm (GA) has been applied to solve the system of transcendental equations [8]. The objective function is to minimize the total harmonic distortion (THD) with the transcendental equations (3-5) are set to be minimization constraints. This should result eliminating the $5^{\text {th }}$ and $7^{\text {th }}$ harmonics. The optimum switching angles of the 7-level CHB-MLI under investigation at 0.8 modulation index are found to be $11.5^{\circ}, 28.7^{\circ}$ and $57.2^{\circ}$ respectively using GAtoolbox in Matlab.

\section{POWER LOSS MODELLING}

When operating power electronics devices that involve switching of semiconductor devices, there are mainly four types of power losses occur during this operation. These types are: 1) Conduction losses, 2) Switching losses, 3) OFF-state losses, and 4) Gate losses. The Off-state and Gate losses are very small and normally neglected. Hence, in this paper, only conduction and switching losses have been considered for the analysis.

Compared to two level inverters, the estimation of inverter losses is a complicated task for multilevel inverters. The usual conventional methods used to calculate the losses in two level inverters, are not suitable to be applied in the case of multilevel inverters. Main reason for this is that in multilevel inverters, each semiconductor devise has different current compared to other devices which implies different losses behavior for each one. This results from having different on-state ratio for each device for one leg during one period of output phase voltage. Furthermore, at higher number of levels, the switching frequency of each device is not the same which add more complexity to the estimation method.

In this paper, a simplified model is proposed for calculating the losses of CHB-MLI precisely. The proposed model uses the method applied in [3] with little modifications. The operating temperature is assumed to be maximum at $150^{\circ}$. The model is based on on-line calculation in which MATLAB-SIMULINK software has been used for the modelling. Mixed load of $\mathrm{R}=60 \Omega$ and $\mathrm{L}=20 \mathrm{mH}$, has been considered for the analysis. The result compared to the case where the load is changing from purely resistive load gradually to purely inductive load. The purpose is to provide a comprehensive study of the inverter losses behavior at different load conditions.

\section{A. Conduction Losses}

For a semiconductor device, the losses which occur while the power device is on the on-state and conducting current, is defined to be the device conduction losses. In CHB-MLI, the conduction loss increases proportionally with the number of cascaded cells. At conduction, the power dissipation can be computed by multiplying the on-state saturation voltage by on-state current.

$p_{\text {Conduction }}=\left|i_{c}\right| \cdot v_{\text {on }}$

The absolute value is taken as the conducting current is always positive for the device. Most of the literatures usually are modelling the on-state voltage by inserting a voltage Vo, representing the voltage drop of the device called threshold voltage, and a resistor ron representing the current dependency in series with the ideal device. The main drawbacks of this modelling approach are [3]:

1) Additional parameters to be added in series with the ideal switches, hence rebuilding the circuit partially. 


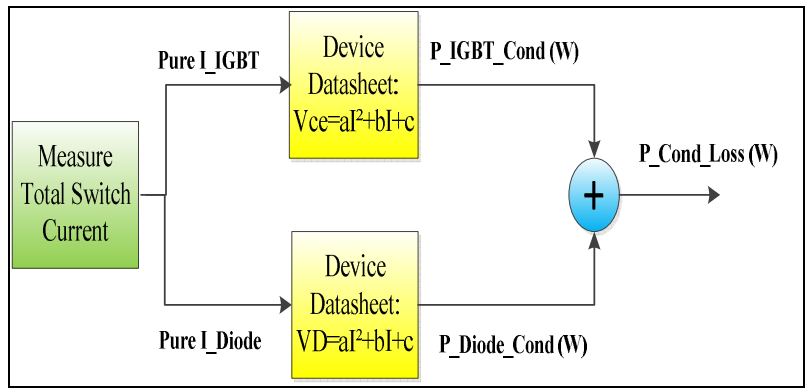

Fig. 4.Conduction losses calculation block.

2) The model might not be accurate as it is not based on actual curves on the device datasheet.

In the proposed model, the conducting losses are computed in much easier and more efficient way. The on-state voltage is represented by a quadratic equation in terms of the on-state current. The quadratic equation is obtained using curve fitting tool based on the actual curves in the device datasheet. For each power device, there will be two quadratic equations, one for the switch and one for the diode. Hence, in the MATLABSIMULINK model, the pure switch current and pure diode current need to be obtained separately. By plotting the onstate device current, the positive portion is representing the pure switch current and the negative portion is representing the pure diode current. Having these currents separated, the conducting losses for the IGBT switch and the diode can be obtained simply by applying the following blocks.

\section{B. Switching Losses}

The switching loss can be defined as the power dissipated during the turn-on and the turn-off switching of the power semiconductor device. It occurs on both the switch and the antiparallel diode. The switching loss is highly proportional to the switching frequency, and hence it contributes substantially to the inverter total loss especially for inverters using SPWM. For the power switch, there are the turn-on loss (Eon) and the turn-off loss (Eoff). On the other hand, for the antiparallel diode, only the turn-off (Erec) loss is considered while the turn-on loss is normally neglected due to fast conducting of the diode when it get forward biased. The turn-on loss is less than $1 \%$ compared to the turn-off loss in modern diodes.

In practice, there are five main factors that affect the behaviour of switching loss which are: current being switched, blocking voltage, junction temperature, gate resistor and wiring stray inductance [3]. The switching loss is considered a major drawback in multilevel inverters causing high increase in cost and reducing efficiency in HVDC applications.

This paper proposes an online estimation of switching losses based on the switching energy curves given in the device datasheet. Energy factor curve $(\mathrm{K})$ is obtained by dividing the energy by switching current. Then Curve fitting tool is used to approximate the energy factor curves by second order polynomials. Multiplying the energy factor curve equation by the switching current should give the energy loss which further multiplied by the switching frequency to get the power loss. In case, the blocking voltage is different, a normalization factor is used to adjust that in the loss calculation. Figure (5) shows the block implemented for the switching loss calculation. The complete block for the proposed modelling of losses calculation in CHB-MLI is presented in Figure (6). Table (1) summarizes the modeling equations of the IGBT switch obtained by curve fitting.

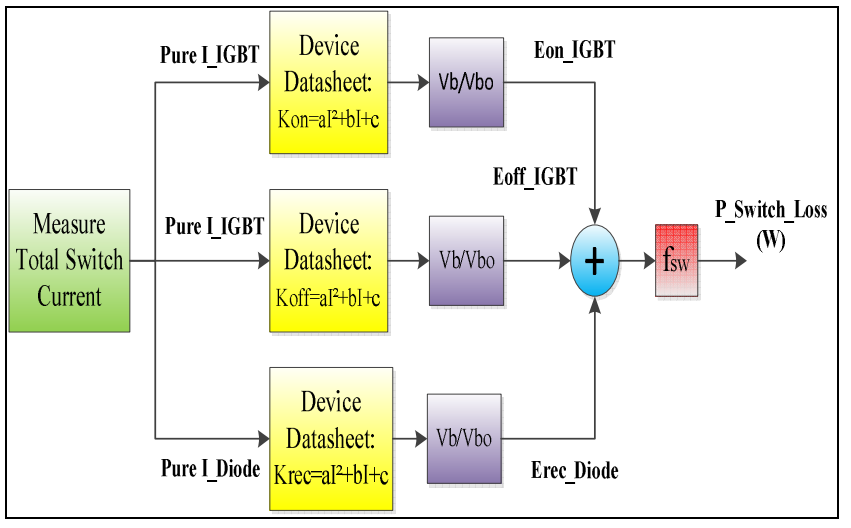

Fig. 5. Switching losses calculation block.

TABLE I

IGBT CURVE FITTED EQUATIONS BASED ON DATASHEET

\begin{tabular}{|c|}
\hline$v_{c e}=-2 \times 10^{-7} I_{c}^{2}+0.0018 I_{c}+0.9661$ \\
\hline$v_{D}=-1 \times 10^{-7} I_{D}^{2}+0.0012 I_{D}+0.7796$ \\
\hline$K_{\text {IGBT-on }}=8 \times 10^{-7} I_{c}^{2}-0.0023 I_{c}+4.016$ \\
\hline$K_{I G B T-\text { off }}=3 \times 10^{-7} I_{c}^{2}-0.0011 I_{c}+3.1584$ \\
\hline$K_{\text {Diode-rec }}=7 \times 10^{-7} I_{D}^{2}-0.0039 I_{D}+6.6546$ \\
\hline
\end{tabular}




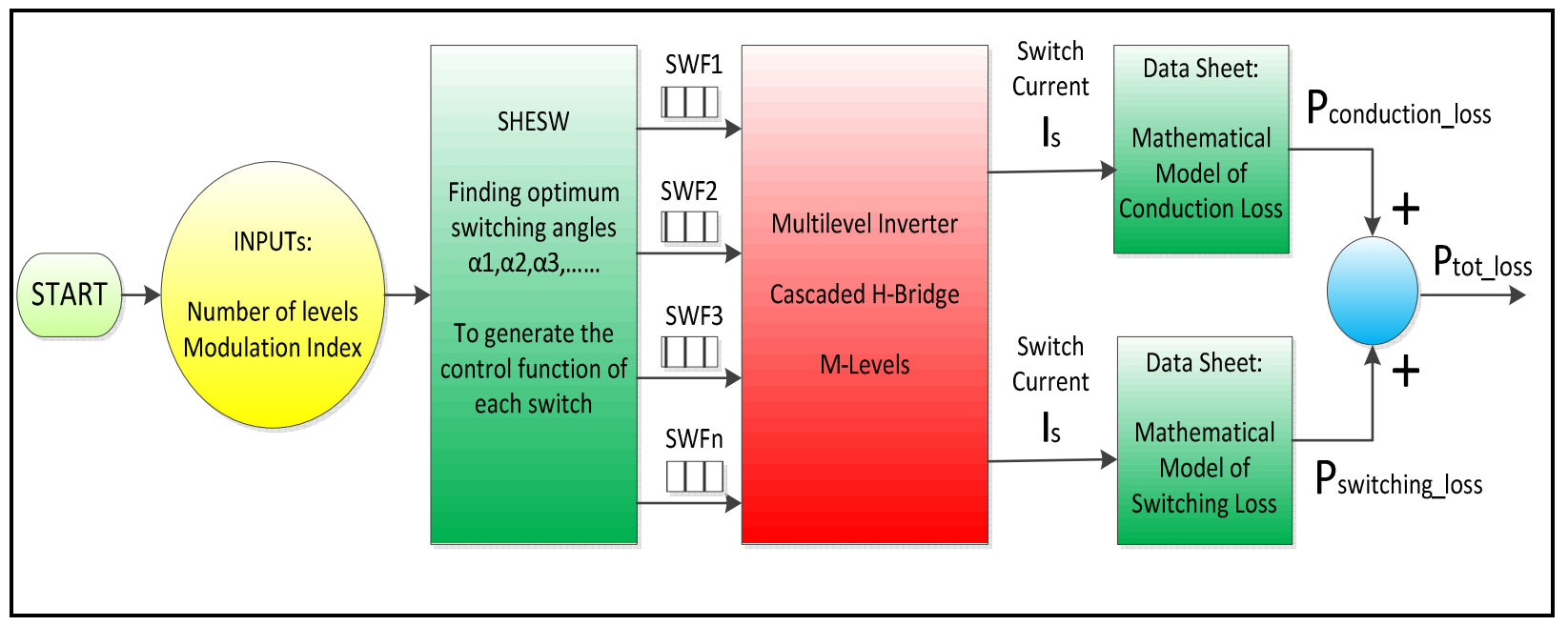

Fig. 6. Basic flow model of proposed methodology for losses calculation in CHB-MLI.

\section{SIMULATION RESULTS AND DISCUSSION}

The proposed online modelling has been implemented for mixed load $(\mathrm{R}=60 \Omega, \mathrm{L}=20 \mathrm{mH})$. The total loss of the inverter was found to be $31.57 \mathrm{~W}$ which represents about $4 \%$ of the total power delivered to load. More than $60 \%$ of the losses comes from conduction losses. This is shown in the pie chart in Figure (7). Looking at the losses in each cell of the inverter, it is clear that cell-1 has the minimum losses compared to cell-2 and cell-3. Figure (8) presents the inverter losses per cell. While most of the switches experience almost the same losses, S5, S7, S9 and S11 consumes much more power loss compared to other switches which is mainly due to their higher switching functions and conduction periods. Comparison of power losses dissipated in each switch is shown in Figure (9)

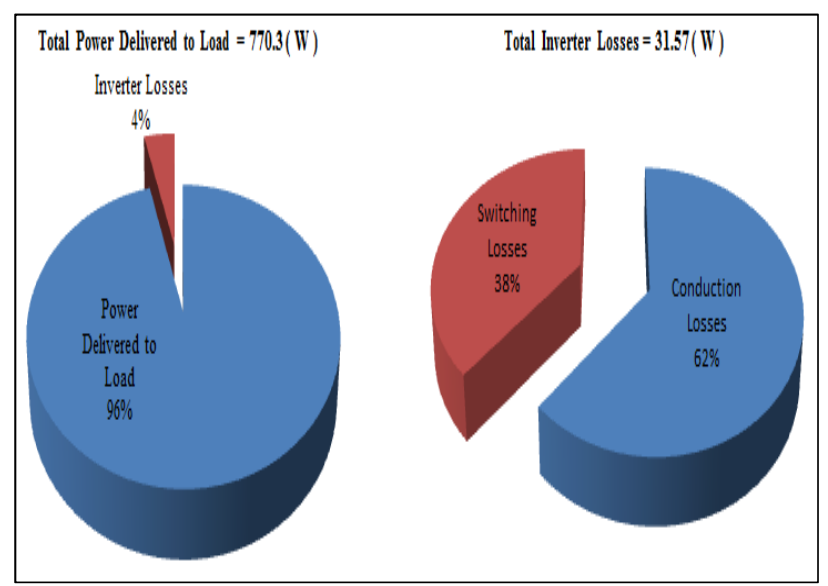

Fig. 7.Inverter total power losses.

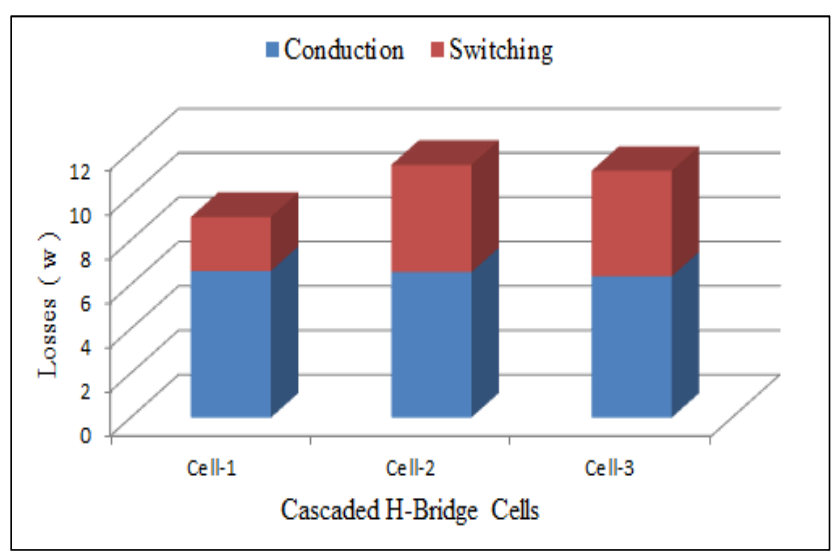

Fig. 8. Switching losses calculation block.

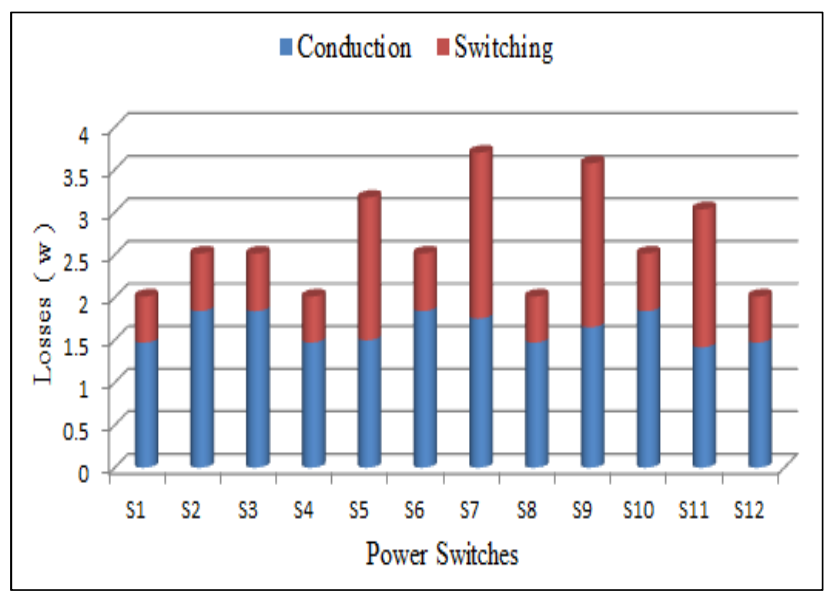

Fig. 9. Switching losses calculation block. 


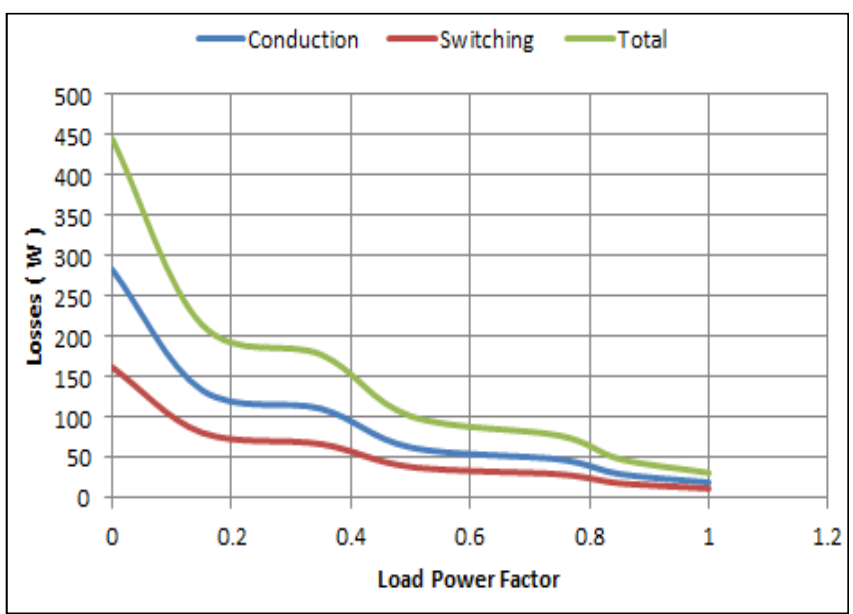

Fig. 10. Inverter losses at different power factors.

In order to investigate the inverter power losses at different power factors, the load has been changed gradually from purely resistive load, mixed RL load to highly inductive load. The losses have been computed at each time at various loads and it is presented here in Figure (10). In all cases the conduction loss is much higher than the switching loss which is mainly due to controlling the inverter using SHE which is a low frequency technique. It is also can be seen that as the load become more inductive, the inverter losses increase significantly. The analysis shows that the inverter losses depends on many factors such as control technique, switching frequency, voltage applied and type of load.

\section{CONCLUSION}

In conclusion, many economic and technical advantages have been achieved after applying multilevel inverter in industrial and utility applications. CHB-MLI is widely applied compared to other topologies. It is critically important to evaluate the losses in multilevel inverters as accurate as possible. Among the many types of losses in multilevel inverters, conduction and switching losses are the most dominant. In this paper, a simple, efficient and easy to implement on-line method for conduction and switching losses calculation in CHB-MLI, has been presented. The model evaluates the losses by calculating the conduction and switching losses in each device separately. Then, adding the losses together. The model use accurate voltage and energy curves as per device datasheet. It was found that, at low switching control, the total inverter losses represents about $4 \%$ of total power delivered by the inverter. About $62 \%$ of total loss occurs while the devices are conducting and only
$32 \%$ of loss comes from switching. The inverter was controlled by SHE which is a low frequency control.

\section{REFERENCES}

[1] N. Mittal, B. Singh, S.P. Singh, R. Dixit and D. Komar, "Multi-Level Inverter: a Literature Survey on Topologies and Control Strategies", $I C P C E S, 2^{\text {nd }}$ International Conference on Power, Control and Embedded Systems, 2012.

[2] J. Ramu, S. Parkash, K. Srinivasu, R. Ram, M. Prasad and Md. Hussain, "Reducing Switching Losses in Cascaded Multilevel Inverters Using Hybrid-Modulation Techniques", IJESE, Vol.2, Issue.4, pp.26-36 April 2012.

[3] Uwe Drofenik, Johann W.Kolar. “ A general Scheme for Calculating Switching- and Conduction Losses of Power Semiconductors in Numerical Circuit Simulations of Power Electronic System", Proc. of the $5^{\text {th }}$ Int. Power Electron. Conference, Niigata, Japan (2005-4).

[4] M.G.H. Aghdam, S.S. Fathi and A. Ghasemi, "The Analysis of Conduction and Switching Losses in Three-Phase OHSW Multilevel Inverter Using Switching Functions", IEE PEDS 2005, Vol. 1, pp. 209-218, 2005.

[5] J. Ramu, S. Parkash, K. Srinivasu, R. Ram, M. Prasad and Md. Hussain, "Comparison between Symmetrical and Asymmetrical Single Phase Seven Level Cascaded H-Bridge Multilevel Inverter with PWM Topology", IJMSE, Vol.3, No.4, pp.16-20 April 2012.

[6] Farzaneh, A.; Nazarzadeh, J. , "Precise Loss Calculation in Cascaded Multilevel Inverters," Computer and Electrical Engineering, 2009. ICCEE '09. Second International Conference on , vol.2, pp.563-568, 28-30 Dec. 2009

[7] C. Govindaraju1 and K. Baskaran, "Performance Improvement of Multiphase Multilevel Inverter Using Hybrid Carrier Based Space Vector Modulation", International Journal on Electrical Engineering and Informatics - Volume 2, Number 2, 2010,pp 137-149.

[8] D. Kumar, S. Pattnaik and V. Singh "Genetic Algorithm Based Approach for Optimization of Conducting Angles in Cascaded Multilevel Inverters", IJERA, Vol. 2, Issue 3, May-Jun 2012, pp.23892395.

[9] Infineon: Datasheet of IGBT module "FZ1500R33HL3 " published at: $\quad$ http://www.infineon.com/cms/en/product/power/igbt/igbtmodule/igbtmodule3300v/FZ1500R33HL3/productType.html?productType $=$ db3a3 $\underline{04443 \mathrm{fc} 5 \mathrm{fe} 1014402996 \mathrm{e} 9 \mathrm{c} 23 \mathrm{a} 2}$ 